\title{
Fuzzy neural network-based robust adaptive control for dynamic positioning of underwater vehicles with input dead-zone
}

\author{
Guoqing Xia, Chengcheng Pang* and Jingjing Xue \\ College of Automation, Harbin Engineering University, Nangang District, Harbin City, Heilongjiang Province, China
}

\begin{abstract}
This paper proposes a design for a robust adaptive controller for the Dynamical Positioning (DP) of underwater vehicles with unknown hydrodynamic coefficients, unknown disturbances and input dead-zones. First, for convenience of controller design, the Multi-Input Multi-Output (MIMO) system is divided into several Single-Input Single-Output (SISO) systems. Next, a Dynamic Recurrent Fuzzy Neural Network (DRFNN) with feedback loops is employed to approximate the unknown portion of the controller, which can greatly reduce the number of neural network inputs. A fuzzy logic dead-zone compensator is designed to cope with the unknown dead-zone characteristics of actuators. The upper bounds of the approximation errors and disturbances of the network, which are often used in existing works, are not necessary in this paper due to the presentation of a special robust compensator. Stability analysis is conducted according to the Lyapunov theorem, and the tracking error is proved to converge to zero. Simulation results indicate that the proposed controller demonstrates good performance.
\end{abstract}

Keywords: Dynamic recurrent fuzzy neural network, underwater vehicle, dynamic positioning, dead-zone, fuzzy compensator

\section{Introduction}

Underwater vehicles have been widely used in marine missions for some time, for such uses as marine science, submarine rescue, data gathering, etc. For some specific tasks, the vehicles are required to precisely maintain positions near structure [3, 20]. Therefore, six Degree of Freedom (DOF) dynamic positioning controllers are necessary for these situations. Due to 6-DOF control issues, the design of controllers is complicated due to the existence of strong coupling character, nonlinear nature, unknown parameters and time-variable disturbances. Therefore, it is a significant and challenging task to design a 6-DOF controller with the ability to handle these factors.

\footnotetext{
${ }^{*}$ Corresponding author. Chengcheng Pang, College of Automation, Harbin Engineering University, No.145 Nantong Street, Nangang District, Harbin City, Heilongjiang Province, China. Tel.: +86 13836191964; Fax: +86 451 82534007; E-mail: pcc_mail@ 163.com.
}

Many researchers have made outstanding contributions to the control issues of underwater vehicles [22]. Mode-based controllers are often utilized in underwater vehicle systems, such as the Lyapunov method, backstepping technique, feedback linearization, and some combined methods $[4,18]$. However, most controllers require an accurate mathematical model of the vehicle, which is often difficult to obtain. Adaptive controllers have been developed in many studies to overcome these problems by estimating the unknown and/or slowlyvarying parameters $[8,21]$. An adaptive PD controller for 4-DOF positioning of a Remotely Operated Vehicle (ROV) is discussed to maintain the position of the ROV close to an underwater structure [6], and the disturbing force caused by ocean current, passive arms, and umbilical cables are compensated for by adaptive estimation. Zhu and Gu proposed an MIMO nonlinear adaptive robust controller with estimated unknown hydrodynamic parameters and slowly varying disturbances [12]. 
Approximation-based control methods such as neural networks and fuzzy logic systems are also effective tools to overcome the limitations of the controller [24-26]. These methods are typically utilized to deal with the unknown terms, nonlinear terms or timevarying disturbances. Ishaque described an efficient fuzzy controller which reduced the conventional twoinput fuzzy controller to a single-input fuzzy controller $[13,14]$. The primary advantage of this approach was that rule inferences were greatly reduced and the calculation procedure was simplified. An adaptive fuzzy sliding mode controller with a novel fuzzy adaptation technique was presented for an Autonomous Underwater Vehicle (AUV) in the presence of external disturbances and parameter uncertainties. $\mathrm{Li}$ and Lee proposed an effective control method, in which the uncertain parameters of AUVs were approximated by a single hidden layered neural network [9]. Also they presented a semi-globally stable adaptive controller for diving control of an AUV, with a neural network to compensate for the unknown terms in pitch motion [10]. Wavelet Neural Networks (WNN) have great advantages in dynamic responses and information storing abilities [1, 2]. A WNN-based adaptive robust controller was investigated to resolve the MIMO tracking control system. Further study introduced a Recurrent Wavelet Neural Backstepping Control (RWNBC) scheme including a neural network and a smooth compensator, which was developed for MIMO systems. An adaptive output feedback controller for AUV was proposed by Zhang, and a Dynamic Recurrent Fuzzy Neural Network (DRFNN) was employed to estimate the dynamic uncertain nonlinear mapping [15]. Since there is an internal feedback loop in this type of neural network, the neural network is able to capture the dynamics of a system, requiring fewer network inputs than conventional neural networks. This paper employs a DRFNN approach to deal with the unknown portion in the ideal controller, which includes the uncertain parameters of the vehicles.

Robust controllers are well known for their immunity to disturbances, and have been widely used in underwater vehicles $[5,11,16]$. Robust terms were often employed to handle the approximation errors of neural networks, external disturbances, and estimation errors of adaptive controllers. However, the upper bounds of the signals were necessary to most existing works, which is difficult to implement in actual systems.

Additionally, from a practical perspective, saturation and dead-zones of the actuators often exist, which will affect the performance of the DP system [19, 23]. This type of vehicle often uses thrusters as actuators, and thrusters have certain inherent nonlinear characteristics such as saturation and dead-zones. However, many control strategies ignored the saturation and dead-zones, due to their different control objectives. As to the DP system under investigation, the speed of the underwater vehicle of the system usually works at low or zero speeds. Also, the existence of the pre-filter will not cause large changes in the error vector, so that the controller outputs will barely reach saturation. Therefore, in this paper, only the effect of the dead-zone will be taken into account.

In current studies, the most common problems in the existing methods of underwater vehicles are as follows: a) AUV controllers are typically designed according to 3-DOF motion. Six-DOF control is more complicated and presents additional challenges; $b$ ) The derivation of the transform matrix is used in many controllers, which is very complex, and the derivation is difficult to obtain for the 6-DOF system; c) In some research, robust terms are used to suppress or offset the effect of jamming, and the upper limits of the disturbances are customarily necessary; d) Most controllers can ensure that the tracking error converges to a neighborhood of zero, which cannot satisfy the precision demands; e) The dead-zone of the actuator was not taken into account in many DP systems.

The primary contribution of this paper is to propose a DRFNN-based adaptive robust controller for 6-DOF underwater vehicles. The controller can be designed without explicit knowledge about the model of the vehicle, and the derivation of the transform matrix. A feed-forward fuzzy compensator is designed to eliminate the effects caused by dead-zones. Additionally, a special robust controller is developed to handle the approximation error of the neural network, estimation error and equivalent disturbances. The bounds of the disturbances are not necessary by utilizing the robust compensator, and the error can converge to zero.

The structure of this paper is organized as follows. Section II describes the control problem of underwater vehicles subject to uncertain terms and disturbances. Section III presents the design of the dynamic recurrent fuzzy neural network-based controller, a fuzzy dead-zone compensator and a robust controller. In section IV, simulation studies are described to verify the effectiveness of the proposed control scheme. Finally, conclusions are made in the final section. 


\section{Problem formulation}

\subsection{Underwater vehicles' kinematics and dynamics}

For the underwater vehicle positioning system, the kinematics and dynamics can be described by the following equations:

$$
\begin{aligned}
& \dot{\boldsymbol{\eta}}=\mathbf{J}(\boldsymbol{\eta}) \boldsymbol{v} \\
& \mathbf{M} \dot{\boldsymbol{v}}+\mathbf{C}(\boldsymbol{v}) \boldsymbol{v}+\mathbf{D}(\boldsymbol{v}) \boldsymbol{v}+\mathbf{G}(\boldsymbol{\eta})=\boldsymbol{\tau}+\Delta f
\end{aligned}
$$

where $\eta=[x, y, z, \phi, \theta, \psi]^{\mathbf{T}} \in \mathfrak{R}^{6 \times 1}$ is the position and attitude vector of the vehicle with 6-DOFs in the earth-fixed frame; $v=[u, v, w, p, q, r]^{\mathrm{T}} \in \mathfrak{R}^{6 \times 1}$ represents the velocities in the body-fixed frame; $\tau \in$ $\Re^{6 \times 1}$ is the input vector, which include the forces and moments produced by thrusters; $\mathbf{M}=\mathbf{M}_{\mathbf{A}}+\mathbf{M}_{\mathbf{R B}}$ is the system inertial matrix,; $\mathbf{C}(\boldsymbol{v})$ is the Coriolis and centripetal matrix; and $\mathbf{C}(\boldsymbol{v})=\mathbf{C}_{\mathbf{A}}(\boldsymbol{v})+\mathbf{C}_{\mathbf{R B}}(\boldsymbol{v}) \in \mathfrak{R}^{6 \times 6}$. The hydrodynamic damping matrix $\mathbf{D}(\boldsymbol{v}) \in \mathfrak{R}^{6 \times 6}$ can be divided into linear and nonlinear parts. $\mathbf{G}(\boldsymbol{\eta}) \in \mathfrak{R}^{6 \times 6}$ represents the gravitational forces and moments; $\Delta f \in$ $\Re^{6}$ is a vector of bias forces and moments and consists of slowly-varying environmental disturbances and unmolded terms; And $\mathbf{J}(\eta) \in \mathfrak{R}^{6 \times 6}$ is the transformation matrix, described as follows:

$$
\mathbf{J}(\boldsymbol{\eta})=\left[\begin{array}{cc}
\mathbf{J}_{\mathbf{1}}(\boldsymbol{\eta}) & \mathbf{0} \\
\mathbf{0} & \mathbf{J}_{\mathbf{2}}(\boldsymbol{\eta})
\end{array}\right]
$$

where $\mathbf{J}_{\mathbf{1}}(\boldsymbol{\eta})$ and $\mathbf{J}_{\mathbf{2}}(\boldsymbol{\eta})$ are complex functions of Euler angles $\phi, \theta, \psi$. By defining $\mathbf{y}=\boldsymbol{\eta}=\left[y_{1}, \ldots, y_{n}\right]^{\mathbf{T}}$, system (1) can be rewritten as follows:

$\ddot{\mathbf{y}}=\left[f_{1}(x) \cdots f_{n}(x)\right]^{T}+G(x)\left[\tau_{1} \cdots \tau_{n}\right]^{T}+\left[d_{1} \cdots d_{n}\right]^{T}$

where $x=\left[\boldsymbol{\eta}^{\mathrm{T}} \dot{\boldsymbol{\eta}}^{\mathrm{T}}\right]^{\mathrm{T}}$ and is equivalent to:

$$
\begin{aligned}
\ddot{y}_{i} & =f_{i}(x)+g_{i 1}(x) \tau_{1}+\cdots+g_{i i}(x) \tau_{i}+\cdots+d_{i} \\
(i & =1, \ldots n)
\end{aligned}
$$

Choose $\tau_{i}$ as the primary input value for the $\mathrm{i}$-th sub-system. Then, the sub-system can be rewritten as follows:

$$
\left\{\begin{array}{l}
\ddot{y}=f_{i}(\mathrm{x})+g_{i i}(\mathrm{x}) \tau_{i}+d_{s i} \\
d_{s i}=\sum_{j=1, j \neq i}^{n} g_{i j}(\mathrm{x}) \tau_{j}+d_{i}
\end{array}\right.
$$

where $d_{s i}$ is the equivalent disturbance of the i-th subsystem. Therefore, the MIMO system is divided into six SISO systems. Assume that the equivalent disturbance of the i-th sub-system satisfies $\left\|d_{s i}\right\| \leq D_{s i}$, and $g_{i i}(x) \geq g_{0}>0$. $D_{s i}$ and $g_{0}$ are unknown constants.

\subsection{Reference trajectory and control objective}

Define the constant vector $\mathbf{y}_{d}=\left[y_{d 1}, \ldots, y_{d n}\right]^{\mathrm{T}}$ as the desired output value of the system. In order to make the differential of $\mathbf{y}_{d}$ always meaningful and improve tracking performance, a smooth reference trajectory $\mathbf{y}_{\text {ref }}=\left[y_{\text {ref } 1}, \ldots, y_{\text {refn }}\right]^{\mathrm{T}}$ for the tracking system will be generated by the pre-filter:

$$
\mathbf{M}_{r} \ddot{\boldsymbol{y}}_{r e f}+\mathbf{D}_{r} \dot{\boldsymbol{y}}_{r e f}+\mathbf{G}_{r} \mathbf{y}_{r e f}=\mathbf{G}_{r} \mathbf{y}_{d}
$$

where $\mathbf{M}_{r}, \mathbf{D}_{r}$, and $\mathbf{G}_{r}$ are positive design matrices.

Remark: In order to ensure that the reference signals $\mathbf{y}_{r e f}, \dot{y}_{\text {ref }}$ and $\ddot{\boldsymbol{y}}_{\text {ref }}$ are all smooth, let $\mathbf{D}_{r}=$ $2 \mathbf{M}_{r} \xi \Omega, \mathbf{G}_{r}=\mathbf{M}_{r} \Omega^{2}$, and $\xi, \Omega>0$ represent diagonal design matrices of relative damping ratios and natural frequencies, and $\xi=\operatorname{diag}\left(\xi_{1}, \xi_{2}, \ldots, \xi_{n}\right), \Omega=$ $\operatorname{diag}\left(\omega_{1}, \omega_{2}, \ldots, \omega_{n}\right)$.

The control objective is to devise a neural networkbased adaptive output feedback controller $\tau$, such that the output signal $\mathbf{y}$ follows a desired reference trajectory $\mathbf{y}_{\text {ref }}$, and the signals in the closed-loop system remain bounded.

\section{Adaptive robust controller design}

In this section, an adaptive robust controller is employed for the DP system of an underwater vehicle.

Considering sub-system (5), the extensional error can be defined as follows:

$$
z_{i}=\dot{e}_{i}+\lambda e_{i}
$$

where $e_{i}=y_{\text {refi }}-y_{i}$ is the tracking error of the i-th subsystem, and $\lambda>0$ is a constant.

Therefore, the $z_{i}$ dynamics can be described as follows:

$$
\begin{aligned}
\dot{z}_{i} & =\ddot{e}_{i}+\lambda \dot{e}_{i} \\
& =\ddot{y}_{\text {refi }}-\ddot{y}_{i}+\lambda \dot{e}_{i} \\
& =-g_{i i}(x) \tau_{i}+a_{i}\left(\dot{y}_{\text {refi }}, \ddot{y}_{r e f i}\right)+b_{i}\left(x, \dot{y}_{i}\right)-d_{s i}
\end{aligned}
$$


where

$$
\begin{aligned}
& a_{i}\left(\dot{y}_{\text {refi }}, \ddot{y}_{r e f i}\right) \\
& \quad=\ddot{y}_{r e f i}+\lambda \dot{y}_{r e f i}, b_{i}\left(x, \dot{y}_{i}\right)=-f_{i}(x)-\lambda \dot{y}_{i} .
\end{aligned}
$$

\subsection{Controller design without disturbances}

An ideal controller is derived in this section for the $z_{i}$ dynamics under the assumption $d_{s i}=0$ :

$$
\begin{aligned}
\tau_{i}^{*}= & \lambda K_{i} e_{i}+g_{i i}^{-1}(x)\left(\bar{a}_{i}\left(y_{\text {refi }}, \dot{y}_{\text {refi }}, \ddot{y}_{\text {refi }}\right)\right. \\
& \left.+\bar{b}_{i}\left(x, y_{i}, \dot{y}_{i}\right)\right),(i=1,2 \cdots n)
\end{aligned}
$$

where $\quad \bar{a}_{i}\left(y_{\text {refi }}, \dot{y}_{\text {refi }}, \ddot{y}_{\text {refi }}\right)=a_{i}\left(\dot{y}_{\text {refi }}, \ddot{y}_{\text {refi }}\right)+g_{i i}(x)$ $K_{i} \dot{y}_{\text {refi }} \quad\left(K_{i}>0\right), \quad$ and $\quad \bar{b}_{i}\left(x, y_{i}, \dot{y}_{i}\right)=b_{i}\left(x, \dot{y}_{i}\right)-$ $g_{i i}(x) K_{i} \dot{y}_{i}$.

Considering the Lyapunov function candidate as follows:

$$
V^{*}=0.5 z_{i}^{2}
$$

Therefore, the derivative of function along the trajectory can be written as follows:

$$
\begin{aligned}
\dot{V}^{*} & =z_{i} \dot{z}_{i}=-g_{i i}(x) K_{i} z_{i}\left(\dot{y}_{\text {refi }}-\dot{y}_{i}+\lambda e_{i}\right) \\
& \leq-g_{0} K_{i} z_{i}^{2} \leq 0
\end{aligned}
$$

Thus, the asymptotic result is $\lim _{t \rightarrow \infty} z_{i}=0$. Therefore, controller (9) can make the extensional error $z_{i}$ converge to zero.

Considering the ideal controller, the second term of the right side includes vehicle parameters. Let:

$$
u_{1}^{*}=g_{i i}^{-1}(x)\left(\bar{a}_{i}\left(y_{\text {refi }}, \dot{y}_{\text {refi }}, \ddot{y}_{\text {refi }}\right)+\bar{b}_{i}\left(x, y_{i}, \dot{y}_{i}\right)\right)
$$

However, for 6-DOF underwater vehicles, it is often impractical to obtain all values in the system matrix, and the modelling error cannot be ignored. For these reasons, a DRFNN is employed to approximate the unknown function in controller, so the controller can be rewritten as:

$$
\tau_{i}=\lambda K_{i} e_{i}+\hat{u}_{r i}+\varepsilon
$$

where $\hat{u}_{r i}$ is the output of DRFNN (which will be designed later), and $\varepsilon$ is the approximate error.

\subsection{Dynamic recurrent fuzzy neural network}

Different to traditional fuzzy neural networks, DRFNN with memory elements and an internal feedback loop can capture the dynamic response of a system. The derivatives $\dot{y}$ that cannot be obtained directly will

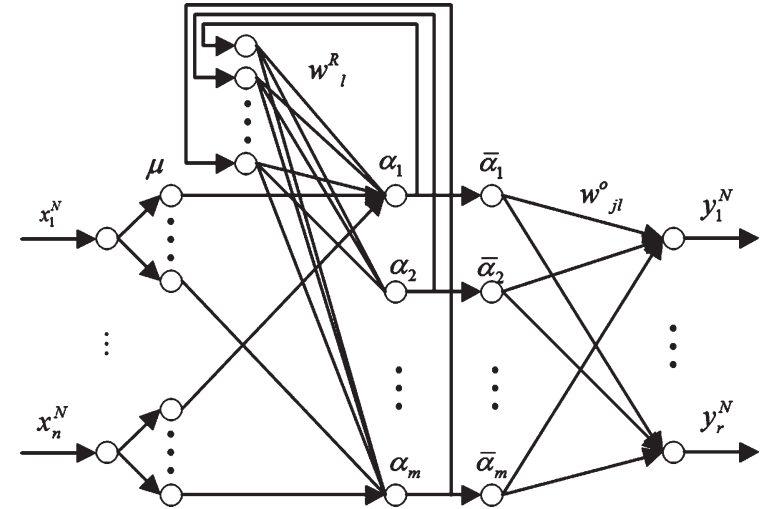

Fig. 1. The structure of DRFNN.

not be used as one of the input vector. For a neural network with $m$ inputs and $n$ outputs, the structure is introduced as follows, and as shown in Fig. 1.

The DRFNN comprises five layers: input layer, fuzzy layer, fuzzy rule layer, normalized layer and output layer. The variables $x^{N}, y^{N}$ are the input and output vectors of the neural network, respectively, and:

$$
y^{N}=\mathbf{W}^{T} \Phi\left(x^{N}\right)
$$

where $\Phi\left(x^{N}\right)=\left[\bar{\alpha}_{1}, \bar{\alpha}_{2}, \ldots \bar{\alpha}_{m_{l}}\right]^{T}$. Therefore:

$$
y_{j}^{N}=\sum_{l=1}^{m} w_{j l}^{o} \bar{\alpha}_{l}, j=1,2, \ldots r
$$

where $w_{j l}^{o}$ is the output weighting value, $m_{l}$ is the number of fuzzy rulers, and the normalized value can be obtained as follows:

$$
\bar{\alpha}_{l}=\alpha_{l} /\left(\sum_{j=1}^{m} \alpha_{j}\right), l=1,2, \ldots m_{l}
$$

where:

$$
\begin{aligned}
& \rho_{l}^{a}(k)=\mu_{1}^{i_{1}}\left(x_{1}^{N}(k)\right) \ldots \mu_{n}^{i_{n}}\left(x_{n}^{N}(k)\right) \\
& \alpha_{l}(k)=\left(1+e^{-\mu\left(\sum_{l=1}^{m} w_{l}^{R} \alpha_{l}(k-1)+z_{l}^{\alpha}(k)-0.5\right)}\right)^{-1}
\end{aligned}
$$

$\mu_{1}^{i}(\bullet)$ is a Gaussian function used to calculate the membership function of every linguistic variable.

As shown by the above Equation (17), every rule application degree at time $k$ not only contains the value at the current time, i.e. $\rho_{l}^{a}(k)$, but also contains the application degree at time $k-1$.

The weight values $w_{l}^{R}$ and $w_{j l}^{o}$ are collected into $\mathbf{W}^{\mathbf{R}}$ and $\mathbf{W}$. Define the parameter vector of the neural network as follows:

$$
\mathrm{E}=\left[\mathbf{W}_{i}^{T} \mathbf{W}_{i}^{\mathbf{R} T}\right]^{T}
$$


According to the approximation theorem [7, 17], there exist matrices $\mathbf{W}^{*}, \mathbf{W}^{\mathbf{R} *}$, such that the continuous function on a compact set can be approximated by recurrent wavelet of the neural work, as follows:

$$
u_{r i}^{*}=g_{i i}^{-1}(x)\left(\bar{a}_{i}+\bar{b}_{i}\right)=y_{D R F N N}\left(x^{N}, \mathbf{E}^{*}\right)+\varepsilon
$$

where $\|\varepsilon\|<\varepsilon_{N}$, and $\varepsilon_{N}$ is the unknown upper bound of the reconstruction error $\varepsilon$. Since the optimal values are unknown, $\hat{E}$ is sued as the estimation of the optimal values, which will be used in the final controller as follows:

$$
\begin{aligned}
\tau_{i} & =\lambda K_{i} e_{i}+\hat{u}_{r i}+u_{s i} \\
\hat{u}_{r i} & =y_{R W N N}\left(x^{N}, \hat{\boldsymbol{E}}\right)
\end{aligned}
$$

where $u_{s i}$ is the robust term to compensate for the approximate errors, and will be designed later.

\subsection{Adaptation laws}

Define the estimation error vectors as follows:

$$
\tilde{\boldsymbol{E}}=\boldsymbol{E}-\hat{\boldsymbol{E}}
$$

Therefore, the reconstruction error of the RWNN can be written as follows:

$$
\begin{aligned}
\tilde{u}_{r i} & =u_{r i}^{*}-\hat{u}_{r i} \\
& =y_{D R F N N}\left(x^{N}, \boldsymbol{E}^{*}\right)-y_{D R F N N}\left(x^{N}, \hat{\mathrm{E}}\right)+\varepsilon \\
& =\left.\tilde{\mathrm{E}}^{T} \frac{\partial y_{D R F N N}\left(x^{N}, \mathrm{E}^{*}\right)}{\partial \mathrm{E}}\right|_{\mathrm{E}=\hat{\mathrm{E}}}+o\left(\tilde{\mathrm{E}}^{2}\right)+\varepsilon
\end{aligned}
$$

where $o\left(\tilde{\mathrm{E}}^{2}\right)$ are the high order terms, and

$$
h_{i}=o\left(\tilde{\mathrm{E}}^{2}\right)+\varepsilon
$$

Therefore, the reconstruction error $\tilde{u}_{r i}$ can be rewritten as follows:

$$
\tilde{u}_{r i}=\left.\tilde{\mathrm{E}}^{T} \frac{\partial y_{R W N N}\left(x^{N}, \mathrm{E}^{*}\right)}{\partial \mathrm{E}}\right|_{\mathrm{E}=\hat{\mathrm{E}}}+h_{i}
$$

For the i-th sub-system, the adaptive laws are defined as follows:

$$
\dot{\mathbf{E}}=\gamma\left(\left.z_{i} \frac{\partial y_{R W N N}\left(x^{N}, \mathrm{E}^{*}\right)}{\partial \mathrm{E}}\right|_{\mathrm{E}=\hat{\mathrm{E}}}-\sigma\left|z_{i}\right| \hat{\mathrm{E}}\right)
$$

where $\gamma>0, \sigma>0$ are the adaptive gain.

\subsection{Compensation of unknown dead-zone}

However, when the speed of the vehicle is maintained around zero, especially with small disturbance forces, the engine of the thrusters must switch between forward

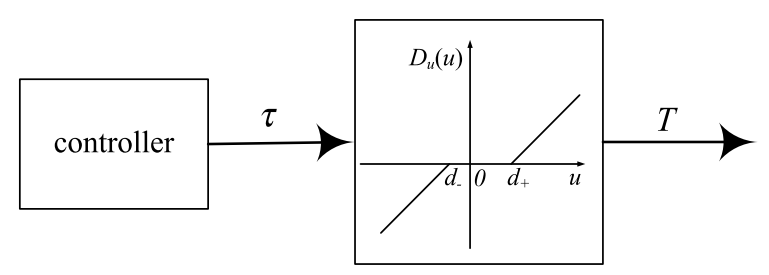

Fig. 2. Dead-zone nonlinearity.

and reverse in order to maintain the vehicle's position. Direct application of the control law mentioned above will cause the motion of the vehicle to shock. Therefore, the effect introduced by the dead-zone of the thrusters should not be ignored. To compensate for the non-symmetric nonlinear dead-zone, a fuzzy logic (FL) feed-forward compensator is designed in this section.

The dead-zone nonlinearity of the actuator is shown in Fig. 2, and it can be written as follows:

$$
T_{i}=D_{u}\left(\tau_{i}\right)=\left\{\begin{array}{cc}
\tau_{i}-d_{i-}, & \tau_{i}<d_{i-} \\
0, & d_{i-} \leq \tau_{i}<d_{i+} \\
\tau_{i}-d_{i+}, & \tau_{i} \geq d_{i+}
\end{array}\right.
$$

where $\tau_{i}$ is the controller output before the dead-zone abd $T_{i}$ is the output of the dead-zone, which represents the real control force acting on the vehicles; and $d_{i-} \in$ $\mathfrak{R}^{-}, d_{i+} \in \mathfrak{R}^{+}$are the unknown and bounded parameters of the dead-zone. For convenience, the dead-zone model can be expressed as follows:

$$
T_{i}=\tau_{i}-\operatorname{sat}_{d}\left(\tau_{i}\right)
$$

where $\operatorname{sat}_{d}(\bullet)$ is the saturation function. From the properties of dead-zones, define the fuzzy rules as follows:

If $\left(\tau_{i} \in X_{+}\left(\tau_{i}\right)\right)$ then $\left(\tau_{F_{i}}=\hat{d}_{i+}\right)$

If $\left(\tau_{i} \in X_{-}\left(\tau_{i}\right)\right)$ then $\left(\tau_{F_{i}}=\hat{d}_{i-}\right)$

where $X_{+}(\cdot)$ and $X_{-}(\cdot)$ are the membership functions (MFs) defined as follows:

$$
X_{+}\left(\omega_{i}\right)=\left\{\begin{array}{l}
0, \tau_{i}<0 \\
1, \tau_{i} \geq 0
\end{array}, X_{-}\left(\omega_{i}\right)= \begin{cases}1, & \tau_{i}<0 \\
0, & \tau_{i} \geq 0\end{cases}\right.
$$

Let $d_{i}=\left[d_{i+}, d_{i-}\right]^{\mathrm{T}}, \mathbf{X}\left(\tau_{i}\right)=\left[X_{+}\left(\tau_{i}\right), X_{-}\left(\tau_{i}\right)\right]^{T}$. The forward compensator is designed here to compensate for the effect of the dead-zone, and make $\tau_{i} \approx T_{i}$. The new control input $\bar{\tau}_{i}$ can be written as follows:

$$
\bar{\tau}_{i}=\tau_{i}+\tau_{F i}, \text { and } \tau_{F i}=\hat{d}_{i} \mathbf{X}\left(\tau_{i}\right)
$$


Therefore, when using a compensator, the real control force $T_{i}$ is expressed as follows:

$$
T_{i}=D_{u}\left(\bar{\tau}_{i}\right)=\tau_{i}-\tilde{d}_{i}^{T} \bar{\kappa}+\tilde{d}_{i}^{T} \delta
$$

where $\Upsilon=u_{S}\left(\tilde{d}_{i}^{T}\right) \mathbf{X}\left(\tau_{i}\right) \Lambda\left(\tau_{i}\right) ; u_{S}(\bullet)$ is a unit step function; $\tilde{d}_{i}=d_{i}-\hat{d}_{i}$ is the estimation error; and $\Lambda\left(\tau_{i}\right)$ is a piecewise function, described as follows:

$$
\Lambda\left(\tau_{i}\right)=\left\{\begin{array}{cc}
0 & \tau_{i}<\tilde{d}_{i-} / \tau_{i}>\tilde{d}_{i+} \\
1-\tau_{i} / \tilde{d}_{i-} & \tilde{d}_{i-} \leq \tau_{i}<0 \\
1-\tau_{i} / \tilde{d}_{i+} & 0 \leq \tau_{i}<\tilde{d}_{i+}
\end{array}\right.
$$

As shown by the definition, $\|\Upsilon\| \leq 1$. Using Lyapunov's direct method, the adaptive tuning law of the estimated dead-zone widths can be determined as follows:

$$
\dot{\hat{d}}_{i}=\gamma_{d}\left(z_{i} \bar{\kappa}-\sigma_{d}\left|z_{i}\right| \hat{d}_{i}\right)
$$

where $\gamma_{d}>0$ and $\sigma_{d}>0$ are design parameters.

\subsection{Final control scheme}

To obtain the final controller, a robust term should first be designed to handle the errors.

Define the total residual error of the system as follows:

$$
\Delta_{i}=h_{i}+\sigma\|\tilde{\mathrm{E}}\|^{2}+g_{0}^{-1} D_{s i}+\sigma_{d}\left\|d_{i}\right\|^{2}+\left\|\tilde{d}_{i}\right\|
$$

where $\Delta_{i}$ is an unknown slowly-varying bounded function.

A special robust controller $u_{s i}$ is chosen as follows to balance the estimation errors and the disturbances in the system, and make the tracking errors approach zero.

$$
u_{s i}=z_{i}\left|z_{i}\right| \hat{\Delta}_{i} /\left(\left|z_{i}\right|^{2}+\delta_{i}\right)
$$

where

$$
\begin{aligned}
\dot{\hat{\Delta}}_{i} & =\gamma_{\Delta}\left|z_{i}\right| \\
\dot{\delta}_{i} & =-\gamma_{\Delta}\left|z_{i}\right| \hat{\Delta}_{i} /\left(\left|z_{i}\right|^{2}+\delta_{i}\right)
\end{aligned}
$$

where $\gamma_{\Delta}>0$ is the adaptive gain, and $\delta_{i}>0$.

Remark: The existence of $\left|z_{i}\right|=z_{i} \operatorname{sign}\left(z_{i}\right)$ will result in chattering phenomenon when using the robust controller. In order to reduce chattering, the sign function $\operatorname{sign}(\bullet)$ can be replaced by the hyperbolic tangent function $\tanh (\bullet)$. This will not affect the stability of the system.

From the above sub-sections, the final control scheme is obtained, and which is illustrated in Fig. 3.

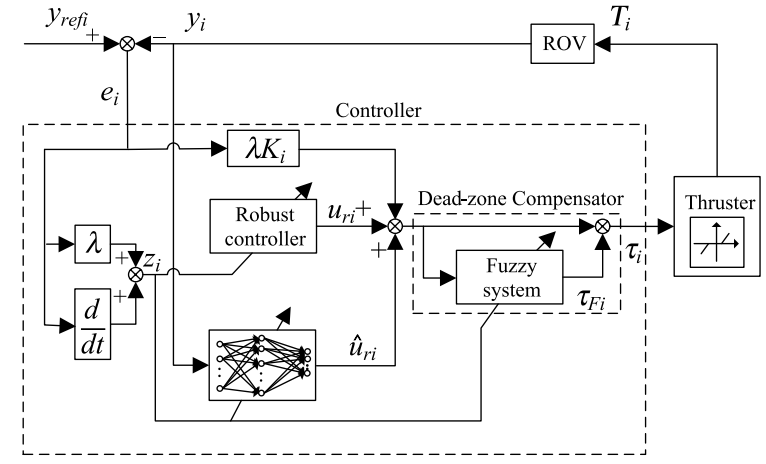

Fig. 3. Architecture of the underwater vehicle control system.

As shown in Fig. $3, \bar{\tau}_{i}$ is the final control law designed for the i-th sub-system, and has the form:

$$
\begin{aligned}
\bar{\tau}_{i} & =\lambda K_{i} e_{i}+\hat{u}_{r i}+u_{s i}+\tau_{F i} \\
\hat{u}_{r i} & =y_{R W N N}\left(x^{N}, \hat{\boldsymbol{E}}\right) \\
u_{s i} & =z_{i}\left|z_{i}\right| \hat{\Delta}_{i} /\left(\left|z_{i}\right|^{2}+\delta_{i}\right) \\
\tau_{F i} & =\hat{d}_{i} \mathbf{X}\left(\tau_{i}\right)
\end{aligned}
$$

Therefore the final control law is comprised of a DRFNN controller $\hat{u}_{r i}$, a dead-zone compensator $\tau_{F i}$ and a robust controller $u_{s i}$. The DRFNN parameters' adaptive laws are chosen as in Equation (25), and the adaptive law of the dead-zone commentator is as shown in Equation (32). The parameters of the robust controller are adjusted according to Equation (35).

\section{Stability analysis}

In this section, the stability of the designed control scheme will be proved. $T_{i}$ is the real control vector acting on the vehicle. Substituting Equation (30) obtains the $z_{i}$ dynamic, as follows:

$$
\begin{aligned}
\dot{z}_{i}= & -g_{i i}(x) T_{i}+a_{i}+b_{i}-d_{s i} \\
= & -g_{i i}(x)\left(\lambda K_{i} e_{i}+\hat{u}_{r i}+u_{s i}-\tilde{d}_{i}^{T} \bar{\kappa}\right)-g_{i i}(x) \tilde{d}_{i}^{T} \delta \\
& +\alpha_{i}+\beta_{i}-g_{i i}(x) g_{i i}^{-1}(x)\left(\bar{a}_{i}+\bar{b}_{i}\right)+g_{i i}(x) u_{r i}^{*}-d_{s i} \\
= & -g_{i i}(x) \tau_{i}^{*}+a_{i}+b_{i}+g_{i i}(x) \tilde{d}_{i}^{T} \bar{\kappa}-g_{i i}(x) \tilde{d}_{i}^{T} \delta \\
& +g_{i i}(x) \tilde{u}_{r i}-g_{i i}(x)\left(u_{s i}-g_{i i}^{-1}(x) d_{s i}\right)
\end{aligned}
$$


Substituting the estimation error $\tilde{u}_{r i}$ into Equation (37) will yield:

$$
\begin{aligned}
\dot{z}_{i}=- & g_{i i}(x) \lambda K_{i} z_{i}+g_{i i}(x)\left(\left.\tilde{\mathrm{E}}^{T} \frac{\partial y_{R W N N}\left(\xi, \mathrm{E}^{*}\right)}{\partial \mathrm{E}}\right|_{\mathrm{E}=\hat{\mathrm{E}}}\right) \\
+g_{i i}(x) \tilde{d}_{i}^{T} \bar{\kappa}- & g_{i i}(x)\left(u_{s i}-h_{i}-g_{i i}^{-1}(x) d_{s i}\right) \\
& -g_{i i}(x) \tilde{d}_{i}^{T} \delta
\end{aligned}
$$

By defining $\tilde{\Delta}_{i}=\Delta_{i}-\hat{\Delta}_{i}$, a Lyapunov function is chosen as follows:

$$
\begin{gathered}
V=0.5 z_{i}^{2}+0.5 g_{i i}(x)\left(\gamma^{-1} \tilde{\mathrm{E}}^{\mathbf{T}} \tilde{\mathrm{E}}+\gamma_{d}^{-1} \tilde{d}_{i}^{T} \tilde{d}_{i}\right. \\
\left.+\gamma_{\Delta}^{-1} \tilde{\Delta}_{i} \tilde{\Delta}_{i}+\gamma_{\Delta}^{-1} \delta_{i} \delta_{i}\right)
\end{gathered}
$$

Taking the time derivative of the Lyapunov function obtains the following:

$$
\begin{aligned}
& \dot{V}=z_{i} \dot{z}_{i}-g_{i i}(x)\left(\gamma^{-1} \tilde{\boldsymbol{E}}^{\mathbf{T}_{\boldsymbol{i}}} \boldsymbol{E}+\gamma_{d}^{-1} \tilde{d}_{i}^{T \dot{2}} d_{i}\right. \\
& \left.+\gamma_{\Delta}^{-1}\left(\tilde{\Delta} \dot{\hat{i}}_{i}+\delta_{i} \dot{\delta}_{i}\right)\right) \\
& =-g_{i i}(x) \lambda K_{i} z_{i}^{2}-g_{i i}(x) z_{i}\left(u_{s i}-h_{i}-g_{i i}^{-1}(x) d_{s i}\right) \\
& +g_{i i}(x) \tilde{d}_{i}^{T}\left(z_{i} \bar{\kappa}-\gamma_{d}^{-1} d_{i}\right)-g_{i i}(x)\left(\gamma_{\Delta}^{-1} \tilde{\Delta} \dot{\Delta}_{i} \Delta_{i}\right. \\
& \left.-\gamma_{\Delta}^{-1} \delta_{i} \dot{\delta}_{i}\right) \\
& +g_{i i}(x)\left(\tilde { \mathrm { E } } ^ { T } \left(z_{i} \frac{\partial y_{R W N N}\left(\xi, \mathrm{E}^{*}\right)}{2}\right.\right. \\
& \left.\left.\left.\partial \mathrm{E}\right|_{\mathrm{E}=\hat{\boldsymbol{E}}}-\gamma^{-l \dot{\boldsymbol{E}}}\right)-z_{i} \tilde{d}_{i}^{T} \delta\right)
\end{aligned}
$$

Substituting the update laws of Equations (25), (32), and (35) into (40), obtains the following.

$$
\begin{gathered}
\dot{V}=-g_{i i}(x) \lambda K_{i} z_{i}^{2}-g_{i i}(x) z_{i}\left(u_{s i}-h_{i}-g_{i i}^{-1}(x) d_{s i}\right) \\
+\sigma_{d}\left|z_{i}\right| g_{i i}(x) \tilde{d}_{i}^{T} \hat{d}_{i}+\sigma\left|z_{i}\right| g_{i i}(x) \tilde{\mathrm{E}}^{T} \hat{\mathrm{E}} \\
-g_{i i}(x)\left(z_{i} \tilde{d}_{i}^{T} \delta+\tilde{\Delta}_{i}\left|z_{i}\right|+\left|z_{i}\right| \hat{\Delta}_{i} /\left(\left|z_{i}\right|^{2}+\delta_{i}\right)\right)
\end{gathered}
$$

Since:

$$
\tilde{\mathrm{E}}^{\mathbf{T}} \hat{\mathrm{E}} \leq \tilde{\mathrm{E}}^{\mathbf{T}}\left(\mathrm{E}^{*}-\tilde{\mathrm{E}}\right) \leq-0.5\|\tilde{\mathrm{E}}\|^{2}+\left\|\mathrm{E}^{*}\right\|^{2}
$$

In the same way, $\tilde{d}_{i}^{T} \hat{d}_{i} \leq-0.5\left\|\tilde{d}_{i}\right\|^{2}+\left\|d_{i}\right\|^{2}$. Therefore:

$$
\begin{aligned}
\dot{V} \leq- & g_{0} \lambda K_{i} z_{i}^{2}-0.5 \sigma_{d} g_{0}\left|z_{i}\right|\left\|\tilde{d}_{i}\right\|^{2} \\
- & 0.5 \sigma g_{0}\left|z_{i}\right|\|\tilde{\mathrm{E}}\|^{2} \\
& +\left|z_{i}\right| g_{i i}(x)\left(\sigma_{d}\left\|d_{i}\right\|^{2}+\sigma\left\|\mathrm{E}^{*}\right\|^{2}\right. \\
& \left.+h_{i}+g_{0}^{-1} D_{s i}+\left\|\tilde{d}_{i}\right\|\right) \\
& -g_{i i}(x)\left(\tilde{\Delta}_{i}\left|z_{i}\right|+\delta_{i}\left|z_{i}\right| \hat{\Delta}_{i} /\right.
\end{aligned}
$$

$$
\begin{aligned}
& \left.\left(\left|z_{i}\right|^{2}+\delta_{i}\right)\right)-z_{i} g_{i i}(x) u_{s i} \\
=- & g_{0} \lambda K_{i} z_{i}^{2}-0.5 \sigma_{d} g_{0}\left|z_{i}\right|\left\|\tilde{d}_{i}\right\|^{2} \\
& -0.5 \sigma g_{0}\left|z_{i}\right|\|\tilde{\mathrm{E}}\|^{2}
\end{aligned}
$$

From the inequality of $\dot{V}$ given by Equation (43), it is demonstrated that $\dot{V}$ is a negative semi-definite function, i.e. $\dot{V} \leq 0$. This implies that $z_{i}, \tilde{\mathbf{E}}, \tilde{\Delta}_{i}, \delta_{i}$ are bounded. Since $V$ is a non-increasing and bounded function, and $\lim _{t \rightarrow \infty} V(t)=V(\infty)$, let:

$$
\begin{aligned}
L\left(z_{i}, \tilde{d}_{i}, \tilde{\mathrm{E}}\right)= & g_{0} \lambda K_{i} z_{i}^{2}+0.5 \sigma_{d} g_{0}\left|z_{i}\right|\left\|\tilde{d}_{i}\right\|^{2} \\
& +0.5 \sigma g_{0}\left|z_{i}\right|\|\tilde{\mathrm{E}}\|^{2}
\end{aligned}
$$

Therefore,

$$
\int_{0}^{\infty} L\left(z_{i}, \tilde{d}_{i}, \tilde{\mathrm{E}}\right) \leq V(0)-V(\infty)<\infty
$$

Since the function $L\left(z_{i}, \tilde{d}_{i}, \tilde{\mathbf{E}}\right)$ is bounded, according to Barbalat's Lemma, the convergence can be guaranteed, i.e., $L\left(z_{i}, \tilde{d}_{i}, \tilde{\mathbf{E}}\right) \rightarrow 0, t \rightarrow \infty$. Furthermore, this implies that $\left|z_{i}\right|,\left|\tilde{d}_{i}\right|$ and $|\tilde{\mathrm{E}}|$ converge to zero as $t \rightarrow \infty$.

\section{Case study}

To validate the proposed DRFNN-based robust adaptive controller, it is assessed in the MATLAB simulation environment with a 6-DOF nonlinear model of a vehicle. The desired position and attitude can be described as $\mathbf{y}_{d}=\left[\begin{array}{llllll}5 & 2 & 5 & 5 & 5 & 5\end{array}\right]^{T}$.

Assume that the dead-zone widths are:

$$
\left[d_{i-}, d_{i+}\right]=[-300,400], i=1,2, \ldots, 6 .
$$

To verify the effectiveness of the controller and the control effect, unpredictable disturbances and parameter uncertainties are introduced. The environmental disturbances acting on the underwater vehicle and the uncertainties of the model can be treated together, as follows:

$$
\Delta f=[200,200,100,100,100,200]^{\mathrm{T}} * \sin (0.05 t)
$$

The pre-filter is utilized to produce a smooth target path for the controller, and the parameter matrices can be chosen as follows: 

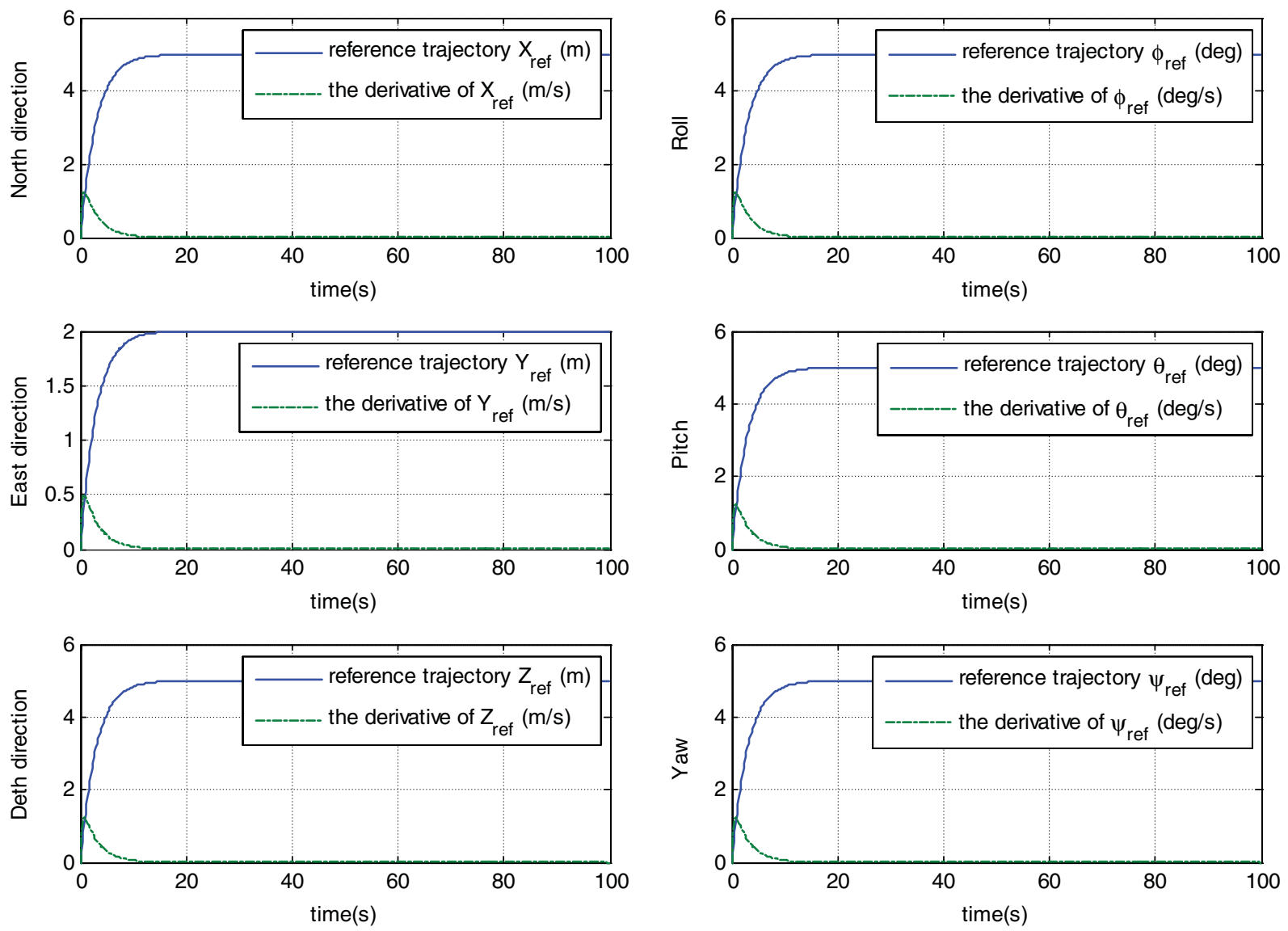

Fig. 4. The reference trajectory generated by the pre-filter.

$$
\begin{aligned}
& \mathbf{M}_{r}=\operatorname{diag}([1,1,1,1,1,1]) \\
& \mathbf{D}_{r}=2 \mathbf{M}_{r} \boldsymbol{\xi} \mathbf{\Omega} ; \mathbf{G}_{r}=\mathbf{M}_{r} \mathbf{\Omega}^{2}, \\
& \boldsymbol{\xi}=\operatorname{diag}([3,3,3,3,3,3]) \\
& \mathbf{\Omega}=\operatorname{diag}([2,2,2,2,2,2])
\end{aligned}
$$

The reference trajectory generated by the pre-filter is shown in Fig. 4.

As shown by the control law in Equation (37), results indicate that the feedback gain $K_{i}$ is similar to the proportional of the PID controller. According to experience and multiple simulation results, an appropriate value of $K_{i}$ can be obtained. The controller parameters were chosen as:

$$
\lambda=0.7 ; K=\operatorname{diag}([300200200300200200])
$$

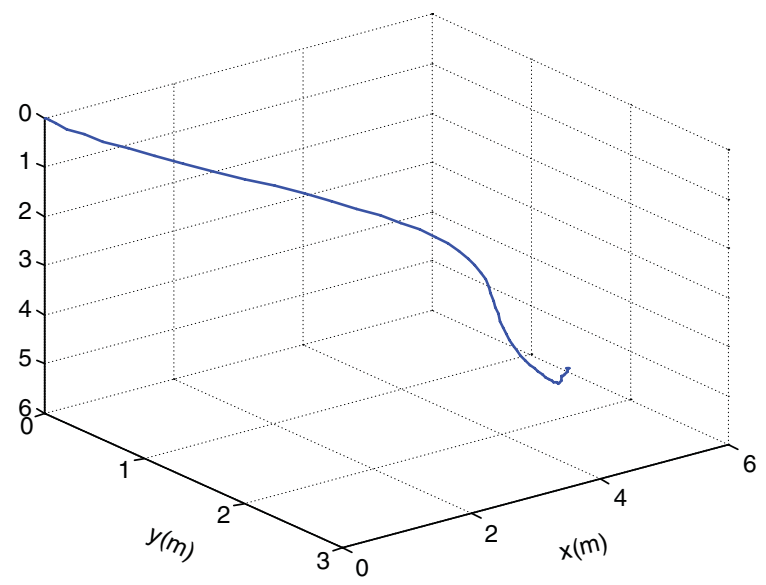

Fig. 5. 3-D trajectory of the underwater vehicle. 
A DRFNN with ten nodes in the product layer is used in the control system; the input vector of the neural network is $\boldsymbol{\xi}=\left[\mathbf{y}^{T}, \mathbf{y}_{\text {ref }}^{T}\right]^{T}$, and the parameters of the weight updating laws are chosen as: $\gamma=20$ and $\sigma=6.2$.

The parameters of the robust controller are chosen as $\gamma_{\Delta}=0.1$. The initial value of $\hat{\Delta}$ is zero, and the initial value of $\delta$ is 0.5 .

The 3-D trajectory of the vehicle is shown in Fig. 5, indicating that the proposed controller can drive the vehicle from the initial position $\mathbf{y}_{0}=\left[\begin{array}{llllll}0 & 0 & 0 & 0 & 0 & 0\end{array}\right]^{T}$ to the given position $\mathbf{y}_{d}$.

Figure 6 shows the curves of the vehicle's position and attitude; the red lines represent the outputs of the pre-filter, and blue lines are the time history of $x, y, z, \phi, \theta, \psi$. As shown by the curves, the output of the vehicle can follow the reference trajectory well. The blue line represents a case with dead-zone com-
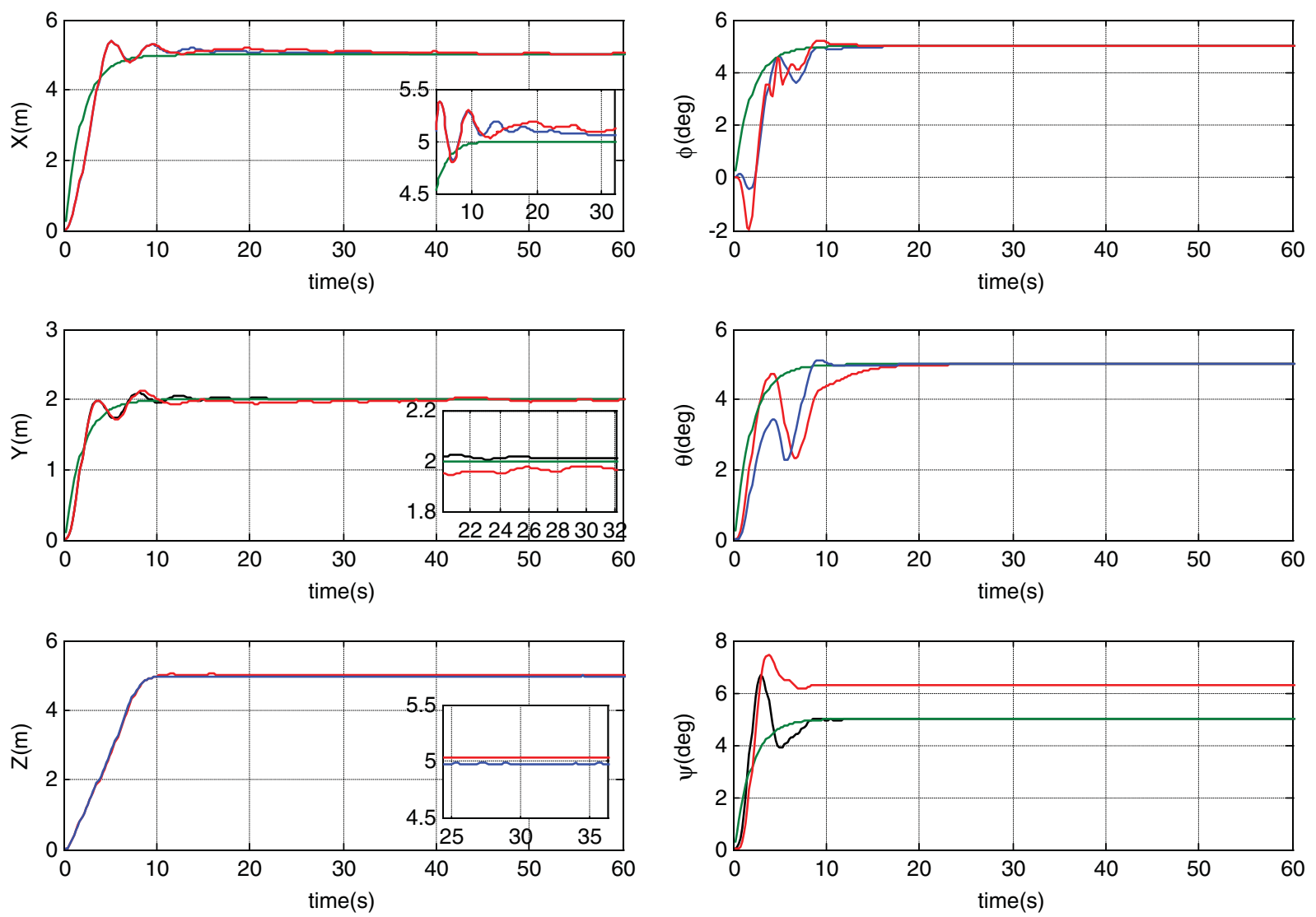

pensation, while the red line represents a case without dead-zone compensation.

Figure 7 shows the time history of the error function with and without dead-zone compensation. As shown by Fig. 7, the error function reaches zero at $15-25$ s using the proposed controller. The performance of the controller is also improved by using dead-zone compensation.

Figure 8 shows the curves of control inputs by using the proposed method. In general, the better tracking precision requires greater efforts, and may cause oscillations in control. As shown in Fig. 5, there are few slight oscillations at the beginning due to the existence of the DRFNN, but the outputs of the thruster are smooth and steady. In addition, the given signals required by the controller are given by the pre-filter, so the errors change smoothly and slowly, ensuring that the magnitude of the thruster can never achieve maximum values.

Fig. 6. The trajectory of the underwater vehicle. 

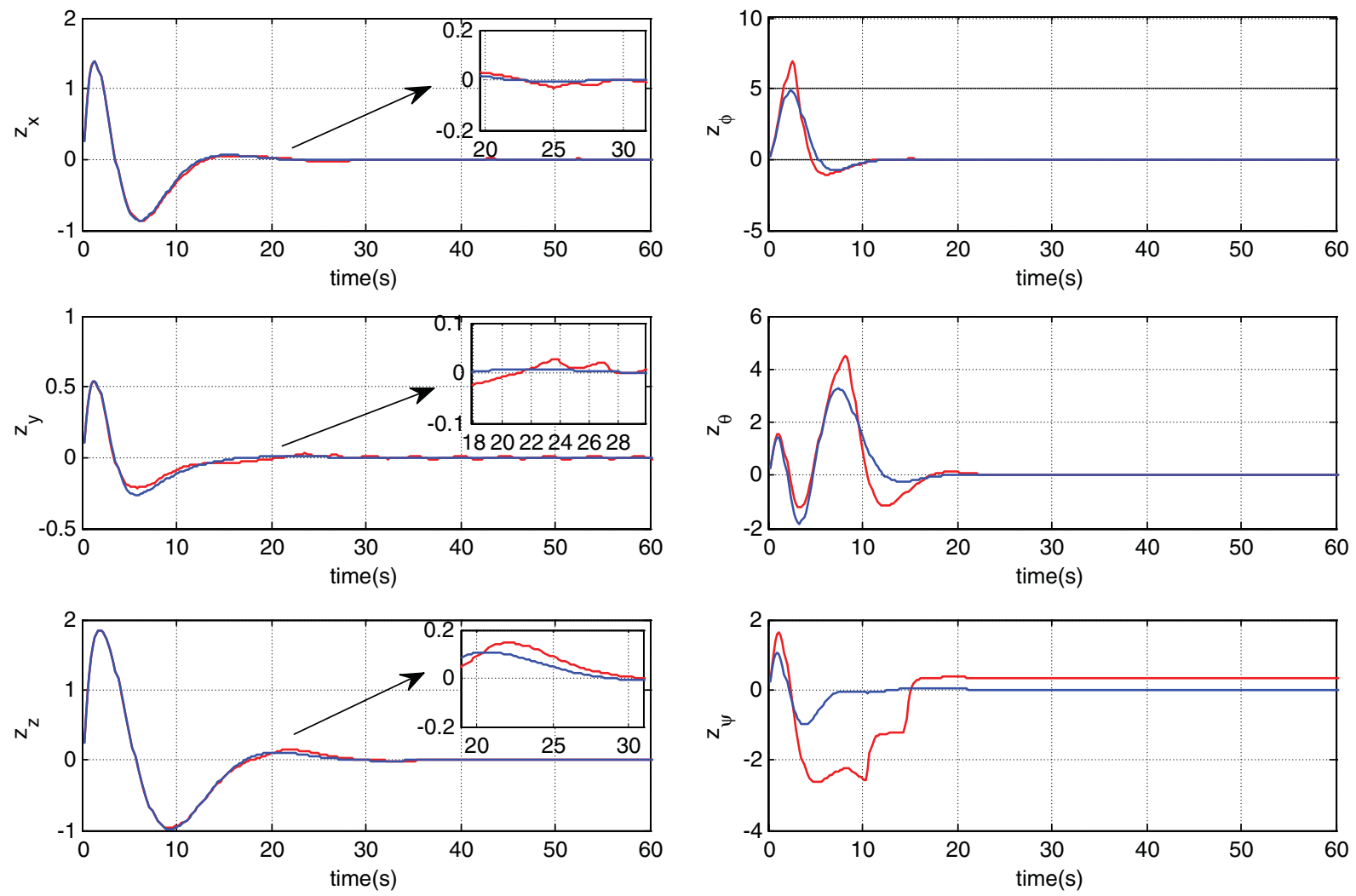

Fig. 7. The time history of the extensional error function.
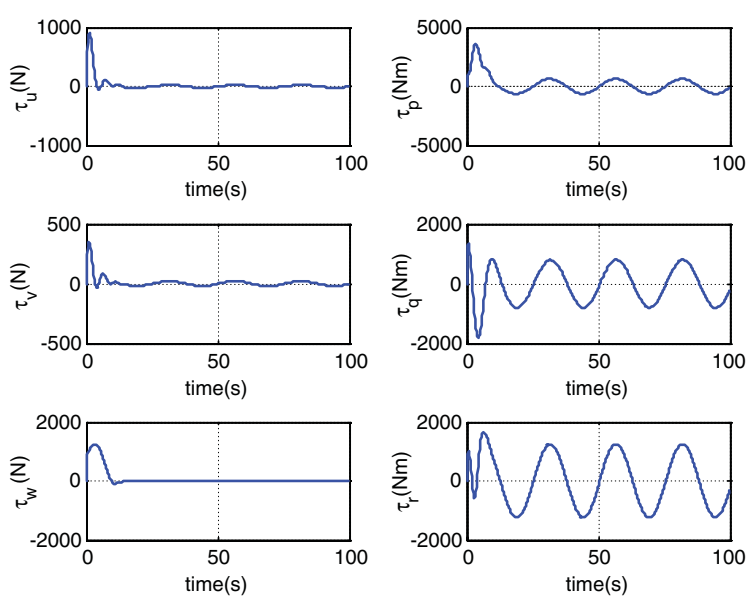

Fig. 8. Control input using the proposed controller.

\section{Conclusions}

This paper investigated the problem of location maintenance for underwater vehicles with parameter uncertainties and unknown external disturbances. At first, the MIMO system was divided into several SISO systems via transformation so that the output of the system was decoupled from the control input. Next, A DRFNN approach is used to estimate the unknown term in the ideal controller, and a special robust controller is designed to compensate for the approximate and equivalent errors. The effect of the dead-zone is eliminated by a fuzzy logic system. The tracking errors can converge to zero. The excellent performance of the aforementioned control scheme is validated by simulation for location maintenance of an underwater vehicle.

\section{Conflict of interests}

The authors declare that there are no conflicts of interest regarding the publication of this article.

\section{References}

[1] C.H. Chen and C.F. Hsu, Recurrent wavelet neural backstepping controller design with a smooth compensator, Neural Computing and Applications 19(7) (2010), 1089-1100. 
[2] C.H. Chen, Wavelet-based adaptive robust control for a class of MIMO uncertain nonlinear systems, Neural Computing and Applications 21(4) (2012), 747-762.

[3] E. Conrado de Souza and N. Maruyama, Intelligent UUVs: Some issues on ROV dynamic positioning, IEEE Transactions on Aerospace and Electronic Systems 43(1) (2007), 214-226.

[4] G. Zhang, L. Yu, X. Meng and L. Zhang, Tracking control of under actuated ship based on partial state feedback scheme, Proceedings of 2011 IEEE/ICME International Conference on Complex Medical Engineering, Harbin, 2011, pp. 678-683.

[5] H. Yang and F. Zhang, Robust control of formation dynamics for autonomous underwater vehicles in horizontal plane, Journal of Dynamic Systems, Measurement, and Control 134(3) (2012), 031009.

[6] H. Nguyen Quang and E. Kreuzer, Adaptive PD-controller for positioning of a remotely operated vehicle close to an underwater structure: Theory and experiments, Control Engineering Practice 15(4) (2007), 411-419.

[7] H. Naira, N. Flavio and K. Nakwan, Adaptive output feedback control of uncertain nonlinear systems using single-hiddenlayer neural networks, IEEE Transactions on Neural Networks 13 (2002), 1420-1431.

[8] J.H. Li and P.M. Lee, Design of an adaptive nonlinear controller for depth control of an autonomous underwater vehicle, Ocean Engineering 32(18) (2005), 2165-2181.

[9] J.H. Li and P.M. Lee, Neural net based nonlinear adaptive control for autonomous underwater vehicles, IEEE International Conference on Robotics and Automation 2 (2002), 1075-1080.

[10] J.H. Li and P.M. Lee, A neural network adaptive controller design for free-pitch-angle diving behavior of an autonomous underwater vehicle, Robotics and Autonomous Systems 52(2) (2005), 132-147.

[11] J. Petrich and D.J. Stilwell, Robust control for an autonomous underwater vehicle that suppresses pitch and yaw coupling, Ocean Engineering 38(1) (2011), 197-204.

[12] K.W. Zhu and L.Y. Gu, A MIMO nonlinear robust controller for work-class ROVs positioning and trajectory tracking control, 2011 Chinese Control and Decision Conference, Mianyang, 2011, pp. 2565-2570.

[13] K. Ishaque, S.S. Abdullah, S.M. Ayob, et al., Single input fuzzy logic controller for unmanned underwater vehicle, Journal of Intelligent and Robotic Systems 59(1) (2010), 87-100.
[14] K. Ishaque, S.S. Abdullah, S.M. Ayob, et al., A simplified approach to design fuzzy logic controller for an underwater vehicle, Ocean Engineering 38(1) (2011), 271-284.

[15] L.J. Zhang, X. Qi and Y.J. Pang, Adaptive output feedback control based on DRFNN for AUV, Ocean Engineering 36(9) (2009), 716-722.

[16] L. Lapierre and B. Jouvencel, Robust nonlinear path-following control of an AUV, IEEE Journal of Oceanic Engineering 33(2) (2008), 89-102.

[17] N. Hovakimyan, F. Nardi and A. Calise, A novel observer based adaptive output feedback approach for control of uncertain systems, IEEE Transactions on Automatic Control 147 (2002), 1310-1314.

[18] P.R. Nambisan and S.N. Singh, Multi-variable adaptive backstepping control of submersibles using SDU decomposition, Ocean Engineering 36(2) (2009), 158-167.

[19] R.R. Selmic and F.L. Lewis, Deadzone compensation in motion control systems using neural networks, IEEE Trans Automat Contrl 45(4) (2000), 602-613.

[20] S.M. Savaresi, F. Previdi, D. Alessandro, B. Sergio, et al., Modeling, identification, and analysis of limit-cycling pitch and heave dynamics in ROV, IEEE Journal of Oceanic Engineering 29 (2004), 407-417.

[21] T.Z. Wu and Y.T. Juang, Adaptive fuzzy sliding-mode controller of uncertain nonlinear systems, ISA Transactions 47(3) (2008), 279-285.

[22] T.I. Fossen, Handbook of Marine Craft Hydrodynamics and Motion Control, John Wiley \& Sons, Chichester, West Sussex, UK, 2011.

[23] T.P. Zhang and S.S. Ge, Adaptive dynamic surface control of nonlinear systems with unknown dead zone in pure feedback form, Automatica 44 (2008), 1895-1903.

[24] Y. Pan, H. Yu and M.J. Er, Adaptive neural PD control with semiglobal asymptotic stabilization guarantee, IEEE Trans on Neural Networks and Learning Systems 25 (2014), 2264-2274.

[25] Y. Pan, J.E. Meng, D. Huang and Q. Wang, Adaptive fuzzy control with guaranteed convergence of optimal approximation error, IEEE Transactions on Fuzzy Systems 19(5) (2011), 807-818.

[26] Y. Pan and J.E. Meng, Enhanced adaptive fuzzy control with optimal approximation error convergence, IEEE Transactions on Fuzzy Systems 21(6) (2013), 1123-1132. 